\title{
Distance Learning mit Moodle - Aktuelles aus Österreichs Schulen
}

•robert.schrenk@lernmanagement.at, Zentrum für Lernmanagement, Pädagogische Hochschule OÖ

eingereicht am: 28.04.2020, akzeptiert am: 30.04.2020

Lehrer/innen in Österreich beschäftigen sich schon seit geraumer Zeit mit der Frage, wie digitale Werkzeuge und Medien das Lernen positiv beeinflussen können. Das österreichische Bundesministerium für Bildung, Wissenschaft und Forschung unterstützt dieses Engagement und stellt eigene Lernplattformen auf Basis von Moodle zur Verfügung, nämlich $\underline{\text { eduvidual.at }}$ und lernplattform.schule.at.

Dieser Beitrag beleuchtet daher, welchen Nutzen Moodle für Schulen stiften kann und wie die aktuelle Verbreitung in Österreich einzuschätzen ist.

Keywords: E-Learning, Lernplattformen, Moodle, eEducation

\section{Distance Learning with Moodle - Current situation in Austrian schools}

For a considerable amount of time, Austrian teachers were investigating in how digital tools and media can influence the learning of their students in a positive way. The Austrian federal ministry of Education supports this investigation by providing learning platforms based on Moodle, namely eduvidual.at and lernplattform.schule.at.

This article examines the current dissemination of Moodle in Austrian schools and how this learning management system can foster digital education.

Keywords: e-learning, learning plattforms, moodle, eEducation

\section{Moodle-Anwendungsszenarien in Österreich}

Generell ist zu sagen, dass es sich bei Moodle nicht um eine konkrete Lernplattform handelt, sondern um ein universelles Lernmanagementsystem, das von Organisationen beliebig auf eigener (on-premise) oder fremder Infrastruktur (off-premise) betrieben werden kann, was durch die fortschreitende Verwendung von Cloud-Services mittlerweile die üblichere Variante darstellt. Durch den Betrieb der Software Moodle für eine bestimmte Zielgruppe entsteht erst die jeweilige Lernplattform.

International gesehen ist Moodle ein ausgesprochen weit verbreitetes Lernmanagementsystem. Über die freiwillige Registrierung von Moodle-Plattformen ist auf stats.moodle.org dokumentiert, dass über 153.000 Organisationen aus 240 Ländern eine solche Lernplattform betreiben und damit über 200 Millionen Nutzer/innen beim Lernen unterstützt werden (Moodle PTy 2020).
Neben dem schulischen Umfeld wird Moodle gemäß dieser Aufstellung in Österreich von verschiedensten Institutionen verwendet. Praktisch alle Universitäten und Fachhochschulen betreiben eine Moodle-Plattform, auch Volkshochschulen (VHS), Berufsförderungsinstitute (BFI) und andere private Fortbildungsinstitutionen sind hier vorzufinden.

Für den Betrieb an Schulen haben sich in Österreich ebenfalls unterschiedliche Varianten etabliert, die für die jeweiligen Schulen auch einen unterschiedlichen Aufwand und unterschiedliche Synergieeffekte bedeuten.

Moodle on-premise, also auf eigener Infrastruktur, zu betreiben, bringt die höchste Flexibilität und den höchsten Aufwand mit sich. Die Erfahrung zeigt, dass vor allem Höhere Technische Lehranstalten (HTL) diese Variante wählen. Neben dem Aufwand geht die Schule auch ein Risiko ein, dass die technische Infrastruktur angreifbar wird und entsprechend geschützt werden muss. Außerdem gibt es nur wenige Synergieeffekte mit anderen Schulen. 


\section{On Premise}

Eigene Lösung

- Server an Schule oder

- Webhosting laas

Landeslösungen

Salzburg NMS-Moodle

Vorarlberger Bildungsserver

lernplattform.schule.at

\section{SaaS}

Landeslösungen

Tiroler Bildungsserver

Bundeslösung

eduvidual.at

\section{Aufwand}

Synergie

Daher werden von einigen Schulerhaltern auch zentrale Services angeboten, die den Schulen dieses Risiko abnehmen. Dabei ist zwischen den Varianten „Infrastructure as a Service“ (IaaS) und „Software as a Service“ (SaaS) zu unterschieden. Bei „Infrastructure as a Service" betreibt der Schulerhalter die Server, jedoch erhält jede Schule eine vollwertige MoodleInstanz und muss noch sehr viele administrative Aufgaben erledigen. Außerdem stellt jede dieser Moodle-Plattformen eine Art „Insel“ dar, wodurch eine Zusammenarbeit mit anderen Schulen erschwert wird und kaum Synergieeffekte zutage treten.

Für die IaaS-Lösung hat sich bspw. der Vorarlberger Bildungsserver (VOBS) und das Bildungsministerium beim Projekt „lernplattform.schule.at" entschieden, welches im Jahr 2004 als „Edumoodle“ gestartet worden war.

Beim „Software as a Service“-Modell werden fast alle administrativen Aufgaben zentral ausgeführt. Die hier genannten Moodle-Plattformen etablieren dabei ein schulübergreifendes Konzept. Dadurch können sich die Schulen voll auf die pädagogische Anwendung der Lernplattform konzentrieren und profitieren von gemeinsamen, überregionalen Materialienpools und anderen bereitgestellten Services.

Die SaaS-Lösung wird vom Tiroler Bildungsserver (TIBS) für alle Tiroler Schulen und vom Land Salzburg für eine NMS-Moodle-Plattform genutzt. Auch das Bildungsministerium stellte das eigene Angebot auf eine solche Lösung um und bietet seit 2018 die schulübergreifende Moodle-Plattform eduvidual.at an, die bis Ende 2021 alle Schulen von lernplattform. schule.at übernehmen wird. Diese Moodle-Plattform steht für alle Bundesschulen und Landesschulen kostenfrei zur Verfügung, sofern letztere Mitglied der e-Education Austria-Initiative sind.

Um diese neue Plattform zu entwickeln und die Schulen zu betreuen, hat das Bildungsministerium „den Auftrag zur Einrichtung des Zentrums für Lernmanagement an der Pädagogischen Hochschule Oberösterreich erteilt, um mit dem Lernmanagementsystem Moodle eine gemeinsame, einheitliche Lernplattform für Österreichs Schulen zu schaffen." (BMBWF 2019)

\section{Verbreitung an Schulen und Nutzungs- statistiken}

Bei der Vielzahl an Moodle-Plattformen, die allein in Österreich zur Anwendung kommen, sind fundierte Zahlen schwer zu bekommen. Zumindest für das Bundes-Moodle-Angebot (lernplattform.schule.at und eduvidual.at) lässt sich aber ableiten, wie viele Schulen davon Gebrauch machen.

Die Verbreitung nach Schultypen und Bundesländern fällt dabei sehr heterogen aus. In diesen Statistiken sind eduvidual.at, lernplattform.schule.at und „BundesMoodle" getrennt ausgewiesen, weil Schulen derzeit die beiden Plattformen eduvidual.at und lernplattform. schule.at auch parallel verwenden können. Als BundesMoodle sind jene Schulen gezählt, die mindestens eine

Tab. 1: Bundes-Moodle nach Regionen (Schrenk 2020)

\begin{tabular}{|l|r|r|r|r|r|}
\hline Bundesländer & Anteil & $\begin{array}{c}\text { Schu- } \\
\text { len } \\
\text { gesamt }\end{array}$ & $\begin{array}{c}\text { Bundes- } \\
\text { Moodle }\end{array}$ & $\begin{array}{c}\text { eduvi- } \\
\text { dual.at }\end{array}$ & $\begin{array}{c}\text { lernplatt- } \\
\text { form. } \\
\text { schule.at }\end{array}$ \\
\hline Burgenland & $2,8 \%$ & 286 & 8 & 4 & 6 \\
\hline Kärnten & $10,9 \%$ & 497 & 54 & 32 & 43 \\
\hline Niederösterreich & $9,1 \%$ & 1261 & 115 & 63 & 76 \\
\hline Oberösterreich & $27,9 \%$ & 1110 & 310 & 141 & 271 \\
\hline Salzburg & $37,1 \%$ & 410 & 152 & 136 & 46 \\
\hline Steiermark & $18,0 \%$ & 934 & 168 & 101 & 117 \\
\hline Tirol & $6,1 \%$ & 693 & 42 & 14 & 34 \\
\hline Vorarlberg & $6,2 \%$ & 292 & 18 & 15 & 3 \\
\hline Wien & $30,4 \%$ & 701 & 213 & 138 & 134 \\
\hline Summe & & 6184 & 1080 & 646 & 730 \\
\hline
\end{tabular}


dieser beiden Plattformen nutzen. Die Spalte „Anteil“ bezieht sich daher auf die Anzahl der Schulen im Bundes-Moodle bezogen auf die Gesamtanzahl der Schulen.

Ein auffallend hoher Anteil an Schulen aus Salzburg $(37 \%)$ sowie Wien und Oberösterreich (etwa $30 \%)$ nutzen das Bundes-Moodle-Angebot. Wenige Schulen aus Tirol und Vorarlberg (6\%) sind beim Bundes-Moodle dabei, wobei diese Bundesländer ja mit TIBS und VOBS eigene Plattformen betreiben. Im Burgenland (3\%) wird flächendeckend auf die alternative Plattform lms.at gesetzt.

Tab. 2: Bundes-Moodle nach Schultypen (Schrenk 2020)

\begin{tabular}{|l|r|r|r|r|r|} 
Schultypen & Anteil & $\begin{array}{c}\text { Schu- } \\
\text { len } \\
\text { gesamt }\end{array}$ & $\begin{array}{c}\text { Bundes- } \\
\text { Moodle }\end{array}$ & $\begin{array}{c}\text { eduvi- } \\
\text { dual.at }\end{array}$ & $\begin{array}{c}\text { lernplatt- } \\
\text { form. } \\
\text { schule.at }\end{array}$ \\
\hline Sonstige & $11,4 \%$ & 492 & 56 & 29 & 42 \\
\hline VS & $3,0 \%$ & 3154 & 94 & 70 & 31 \\
\hline HS/NMS/MS & $34,5 \%$ & 1119 & 386 & 254 & 235 \\
\hline Sonderschule & $15,1 \%$ & 265 & 40 & 34 & 8 \\
\hline PTS & $25,6 \%$ & 180 & 46 & 30 & 23 \\
\hline BS & $23,0 \%$ & 152 & 35 & 22 & 28 \\
\hline Gymnasium & $65,4 \%$ & 370 & 242 & 107 & 209 \\
\hline HTL & $25,3 \%$ & 150 & 38 & 22 & 33 \\
\hline HAK & $48,7 \%$ & 119 & 58 & 27 & 52 \\
\hline HUM & $46,4 \%$ & 183 & 85 & 49 & 69 \\
\hline & & 6184 & 1080 & 646 & 730 \\
\hline
\end{tabular}

Auch hier bezieht sich die Spalte „Anteil“ auf die Anzahl der Schulen im Bundes-Moodle bezogen auf die Gesamtanzahl der Schulen. Als Bundes-Moodle sind jene Schulen gezählt, die mindestens eine dieser beiden Plattformen nutzen. Die Verbreitung des BundesMoodle-Angebots unter österreichischen Gymnasien beträgt etwa $65 \%$, jene in den wirtschaftsberuflichen und humanberuflichen Schulen fast $50 \%$. Immerhin mehr als ein Drittel aller Neuen Mittelschulen und Hauptschulen nutzen das Angebot des Bundes-Moodle.

All diese Zahlen lassen jedoch keinen Schluss darauf $\mathrm{zu}$, wie viele Personen innerhalb der Schulen diese Plattformen dann auch tatsächlich verwenden. Im März 2020 wurden dazu vom Bildungsministerium Statistiken veröffentlicht, die zumindest einen Eindruck vermitteln, wie es um das BundesMoodle-Angebot, nämlich lernplattform.schule.at und eduvidual.at, steht.

Dieses Diagramm wurde von Herrn BM Dr. Faßmann in einer Pressekonferenz am 22. März 2020 im öffentlich-rechtlichen Rundfunk ORF der österreichischen Öffentlichkeit gezeigt. Es dokumentiert deutlich den durch COVID-19 ausgelösten Ansturm auf die Lernplattformen (lernplattform.schule.at, eduvidual.at und lms.at) sowie die Materialienpools (digi4school.at und eduthek.at) des Bildungsministeriums. Diese Zahlen vermitteln einen Eindruck davon, wie sehr Schulen Moodle als Stütze zum digitalen Arbeiten verwenden. Die beiden Moodle-Plattformen lernplattform.schule.at und eduvidual.at verzeichneten am 16. März 2020 mit etwa 270000 Besuchen einen größeren Ansturm, mehr als die übrigen Angebote zusammen.

Man muss allerdings festhalten, dass die Kennzahl der „Besuche“ schwer zu interpretieren ist. Folgende Faktoren sind zu berücksichtigen:

1. Für diese Analyse werden die Log-Dateien der Webserver herangezogen. Daher beeinflusst sowohl die Plattform selbst als auch die WebserverSoftware (Apache/Nginx) durch ihre Log-Ein-

\section{Besuche}

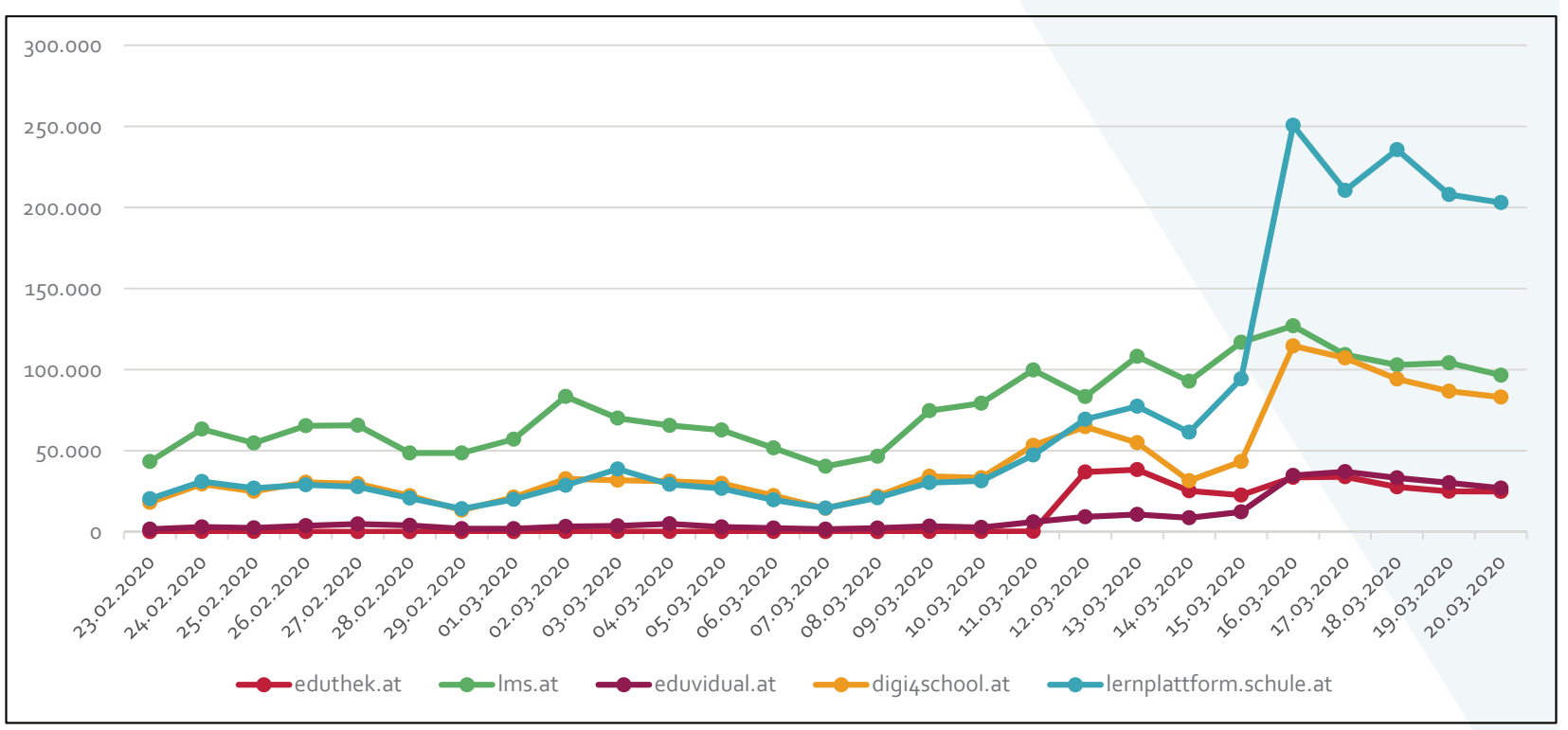


stellungen jene Daten, die zur Zählung überhaupt herangezogen werden können.

2. Jedes Mal, wenn jemand nach einer längeren Pause zu einer Webseite navigiert, gilt das als ein „Besuch“. Manche Personen rufen eine Seite über den Tag verteilt mehrmals auf oder nutzen sie über mehrere Geräte zur gleichen Zeit (Handy, Tablet, Computer, ...).

3. Eine der Plattformen könnte auf einer Vielzahl an schuleigenen Geräten als Startseite konfiguriert sein, wodurch jeder Start eines Browsers als „Besuch“ gezählt werden würde, obwohl der/die Nutzer/in eine andere Seite öffnen wollte.

Zumindest der letztgenannte Effekt lässt sich für die Angaben ab 15. März 2020 ausschließen, da ab diesem Zeitpunkt die Schulen geschlossen waren und ausschließlich auf privaten Endgeräten gearbeitet wurde.

Möglicherweise ist ohnehin die wichtigere Frage, wie viele verschiedene Nutzer/innen überhaupt in einem gewissen Zeitraum auf diese Plattformen zurückgreifen. Das Zentrum für Lernmanagement führt daher seit dem 18. März 2020 genaue Aufzeichnungen darüber, wie viele Nutzerkonten tatsächlich verwendet wurden. Das bedeutet, dass im jeweils genannten Zeitraum mit einem Nutzerkonto zumindest einmal eine Aktivität verzeichnet wurde.

Diese Statistiken zeigen, dass an Wochentagen täglich etwa 85000 Lehrer/innen und Schüler/innen die Moodle-Plattformen nutzen, innerhalb von sieben Tagen sind ungefähr 125000 verschiedene Lehrer/ innen und Schüler/innen aktiv. Auch während der Osterferien (04. April 2020 - 14. April 2020) war eine gewisse Aktivität durch etwa 80000 Nutzer/innen zu verzeichnen.

\section{Moodle als Distance-Learning-Tool}

Zuletzt stellt sich die Frage, was machen diese vielen Nutzer/innen mit Moodle überhaupt?

Das hängt natürlich sehr stark vom persönlichen Unterrichtsstil und den Rahmenbedingungen ab. Die Erfahrung zeigt, dass die persönliche digitale Entwicklung von Lehrenden in gewissen Stufen abläuft.

In der ersten Stufe, wenn Lehrende den digitalen Raum erstmalig entdecken, scheint es ihnen als Mehrwert schon auszureichen, einfach Materialien verteilen zu können. Die exzessive Nutzung dieser Funktion, ohne weitere persönliche Entwicklung, führt aber dazu, dass Moodle zur „PDF-Schleuder“ verkommt, wofür Moodle in der Tat nicht die geeignetste Plattform ist (Jäger, Kieffer, Lorenz \& Nistor 2014). Die Kommunikation erfolgt hier in der Regel unidirektional von den Lehrenden zu den Lernenden und zementiert damit gewissermaßen lehrerzentrierte Methoden.

Daher sollte festgehalten werden, dass die Erschaffer/innen von Moodle genau dieses reine Verteilen von Materialien kritisiert haben und dies sogar einer der Gründe dafür war, Moodle überhaupt zu entwickeln. Moodle stellt das Konzept von „Aktivitäten“ in den Vordergrund, um kooperative, konstruktivistische Lernkonzepte zu ermöglichen, die die Lernenden ins Zentrum des Geschehens rücken (Hillenbrand 2012; Hillenbrand \& Schlindwein 2017).

Viele Lehrer/innen sehen auf der zweiten Stufe dieser Entwicklung vor, dass die Schüler/innen ihnen Dateien zurückschicken können. Durch die Einrichtung eines Rückkanals wird immerhin eine bidirektionale Kommunikation möglich, die die Lernenden ein wenig stärker in den Lernprozess einbindet. Natürlich sind auch hier kollaborative Aufgabenstellungen mög-

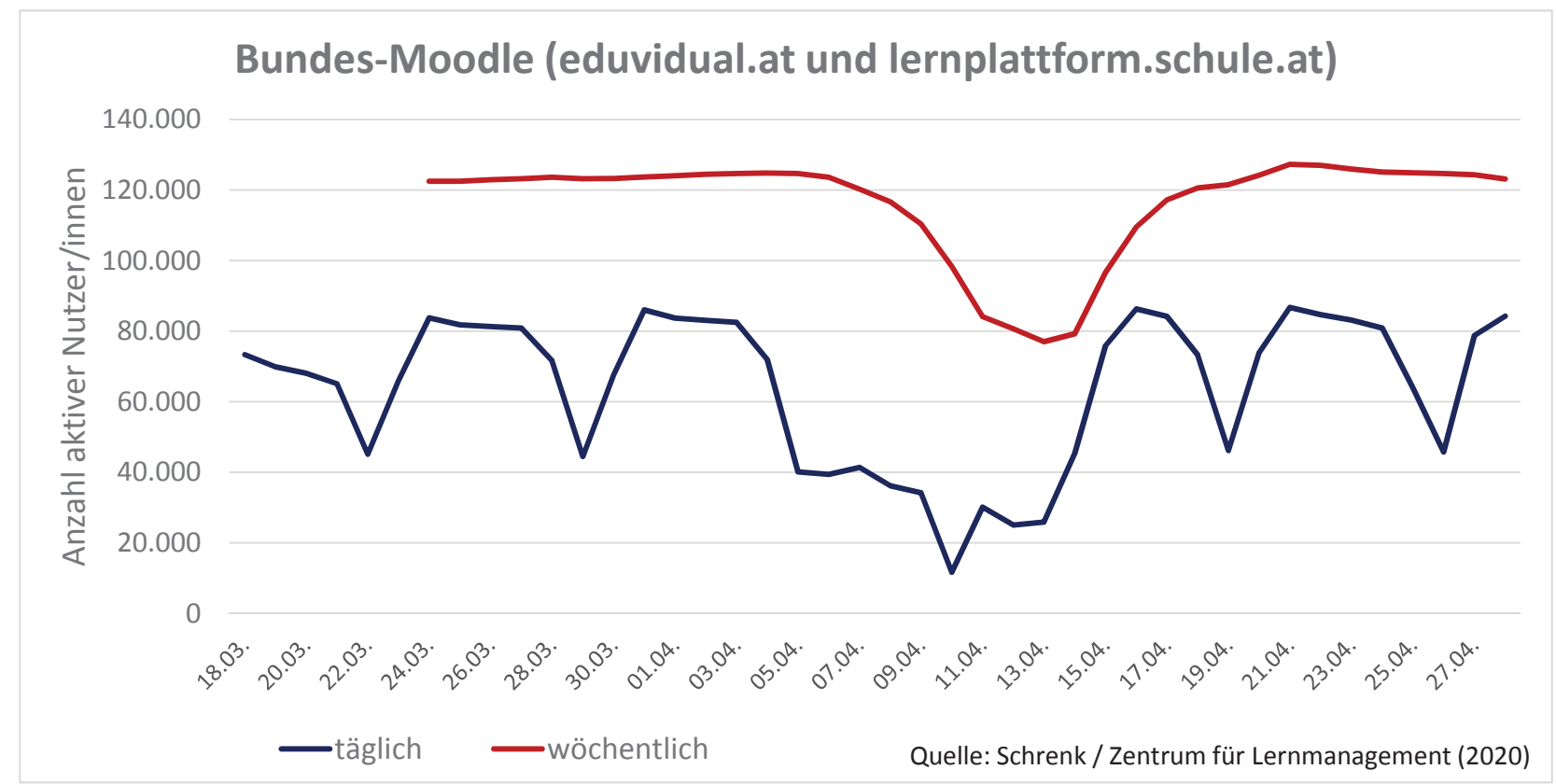


lich, wie bspw. in Form von webbasierten Office-Programmen. Dies stellt aber nicht die Regel dar.

Ein Indiz dafür liefert der wachsende Speicherplatzbedarf des Bundes-Moodle, der sich während der COVID-19-Schulschließungen innerhalb von sechs Wochen fast verdoppelt hat. Dies deutet darauf hin, dass in diesem Zeitraum eine sehr große Anzahl an Lehrer/innen damit begann, Moodle zu verwenden und ihren Unterricht dateibasiert anzulegen. So ist es etwa in Schulen der Primarstufe und Sekundarstufe I durchaus die Regel, dass analoge Arbeitsblätter einfach abfotografiert und abgegeben werden. Das ist keineswegs verwerflich, allerdings sollte das als Zwischenstufe, keinesfalls Endstufe, auf einem Weg zu einer neuen Form von digitalen Aufgaben gesehen werden.

Das SAMR-Modell von Puentedura (2006) stellt eine gute Grundlage dar, um diesen Weg zu erläutern. Gemäß diesem Modell kann eingeschätzt werden, wie sehr Technologie einen Mehrwert für Lernprozesse bedeutet. Diese Stufen beschreibt Puentedura als (1) Substitution (= Ersetzung), (2) Augmentation (= Verbesserung), (3) Modification (= Veränderung), (4) Redefinition (= Neudefinition). Ein Abfotografieren von analogen Arbeitsblättern oder Erfüllen von Aufgaben in einem digitalen Arbeitsblatt stellt eine Substitution, höchstens noch eine Augmentation dar.

Einen höheren Mehrwert durch den Einsatz digitaler Technologie erhält man durch die Veränderung und Neudefinition von Lehraufgaben, die das Potential digitaler Technologien entsprechend ausschöpfen.

Im Fall von Moodle erreicht man solche Aufgaben durch den Einsatz der zuvor genannten „Aktivitäten“. Auch vermeintlich „einfache“ Aktivitäten, wie ein Glossar in Kombination mit Moodle Games, können Lernprozesse auf ein neues Niveau heben und ermöglichen es Lehrerinnen und Lehrern mit einfachsten Mitteln Game-Based-Learning-Sequenzen umzusetzen. Mit Tools wie „Level Up!“ oder „Stash“ lässt sich der Lernprozess durch Gamification ergänzen (Klemke, Eradze \& Antonaci 2018). Vergleichbare Aktivitäten sucht man in Plattformen wie MS-Teams und dgl. vergeblich. Das ist weniger verwunderlich, da der Ursprung von MS-Teams und ähnlichen Plattformen eine Büroumgebung ist, wo es hauptsächlich um Kommunikation und Dokumentenaustausch geht.

Dank des modularen und dynamischen Aufbaus von Moodle (= Modular Object Oriented Dynamic Learning Environment) lassen sich die Moodle-Aktivitäten durch Plug-ins beliebig erweitern, was auch von vielen Unternehmen und Hobby-Entwicklerinnen und -Entwicklern rege genutzt wird. Aktuell sind 1.682 solcher Erweiterungen in der Datenbank von Moodle erfasst, die ein schier endloses Spektrum an Möglichkeiten zur Verfügung stellen.
Darüber hinaus stellt Moodle kein monolithisches System dar, sondern interagiert über Standardschnittstellen mit anderen Systemen (oAuth-Login für Microsoft, Google \& Co, LTI für Austausch mit anderen Lernplattformen, ...). Durch eine Nutzung dieser Schnittstellen lassen sich die Vorteile verschiedener Systeme miteinander kombinieren und eine nahtlose Lernerfahrung, also seamless learning, umsetzen (Schmid \& Schrenk 2017; Mouri, Uosaki \& Ogata 2018).

Am Beispiel von eduvidual.at kann man zeigen, wie bspw. die Planung von MS-Teams-Meetings direkt aus Moodle heraus funktioniert oder Google Drive und Microsoft OneDrive als Datenspeicher eingebunden werden können. Umgekehrt lässt sich ein Moodle-Kurs auch in MS-Teams als Reiter integrieren. Ebenso ist die neue Videoplattform edutube.at, die vom Bildungsministerium gemeinsam mit dem ORF angeboten wird, in eduvidual.at integriert. Lehrer/innen können nahtlos aus dem Fundus dieser Quellen wählen und Materialien einbetten.

Es gibt auch schon Werkzeuge, die eine Synchronisation zwischen MS-Teams-Gruppen und MoodleKursen zulassen, wobei sogar Systemnachrichten aus den Moodle-Kursen im Nachrichtenstrom der MSTeams-Gruppe aufscheinen. Dazu muss man lediglich das Zusatztool „Moodle Dashboard“ von Skooler (2020) in einer MS-Teams-Gruppe aktivieren, sofern $\mathrm{der} / \mathrm{die}$ Moodle-Administrator/innen die entsprechende Konfiguration vorgenommen haben.

Für Schulen in Österreich wäre es daher sehr erstrebenswert, Moodle nicht in Konkurrenz zu Microsoft, Google \& Co zu denken, sondern die sinnvolle Synergie dieser Plattformen zu erkennen und für Schüler/ innen nutzbar zu machen.

\section{Literatur}

BMBWF (2019): IT-Dienstleistungen und Content-Angebote. https://www.bmbwf.gv.at/Themen/schule/zrp/dibi/ itinf/itdienstleistungen.html (23.04.2020)

Hillenbrand, G. (2012): Moodle Philosophie. https://docs. moodle.org/38/de/Philosophie (n. d.)

Hillenbrand, G. \& B. Schlindwein (2017): Moodle Pädagogik. https://docs.moodle.org/38/de/P\%C3\%A4dagogik (n. d.)

Jäger, P., A. Kieffer, A. Lorenz \& N. Nistor (2014): Der Einfluss der didaktischen Gestaltung auf die Akzeptanz und Nutzung von moodle in der Hochschullehre. In: Rummler, K. (Hg.): Lernräume gestalten - Bildungskontexte vielfältig denken. Waxmann, Münster. S. 485-95. http://2014. gmw-online.de/wp-content/uploads/485.pdf (01.05.2020)

Klemke, R., M. Eradze \& A. Antonaci (2018): The Flipped MOOC: Using Gamification and Learning Analytics in MOOCDesign-A Conceptual Approach. In:Education Sciences 8(1). S. 25. https://doi.org/10.3390/educsci8010025 
Kristöfl, R. \& M. Oswald 2020: Auslastungsstatistiken für die IT-Services Eduthek, Digi4School, LMS.at, Eduvidual und Lernplattform.schule.at (Moodle). Wien.

Lieberman, A. (2000): Networks as Learning Communities: Shaping the Future of Teacher Development. In: Journal of Teacher Education 51(3). S. 221-27. https:// doi.org/10.1177/0022487100051003010

Moodle Pty (2020): Moodle-Statistiken. https://stats. moodle.org (29.4.2020)

Mouri, K., N. Uosaki \& H. Ogata (2018): Learning Analytics for Supporting Seamless Language Learning Using E-Book with Ubiquitous Learning System. In: Journal of Educational Technology \& Society 21(2). S. 150-163.

Puentedura, R. (2006): Transformation, technology, and education. http://hippasus.com/resources/tte/ (27.4.2020)
Schmid, S. \& R. Schrenk (2017): Grenzenlos InformellFormell Lernen - Egal Wo Und Wann. Mit Ubiquitärer Technologie Seamless Learning an der FH Burgenland Ermöglichen. In: Trink, A. (Hg.): Pannonia Research Award 2016 Regionale Und Europäische Zukunftsfragen Aus Forschung Und Lehre. Leykam, Graz. S. 97-104. https://fhburgenland.contentdm.oclc.org/digital/collection/p16083coll2/id/439 (01.05.2020)

Schrenk, R. (2020): „Bundes-Moodle“ nach Regionen sowie Bundes-Moodle nach Schultypen. Schriftliche Auskunft am 23.04.2020. Linz.

Skooler (2020): Moodle in Teams. https://skooler.com/ moodle-by-skooler (01.05.2020) 